\title{
Piloting an Online Module for Interprofessional Education to Introduce First-Year Students to Health Behavior Change
}

Michael J. Peeters, PharmD, MEd, BCPS1' John M. Wryobeck, PhD

${ }^{1}$ University of Toledo College of Pharmacy \& Pharmaceutical Sciences; ${ }^{2}$ University of Toledo College of Medicine \& Life Sciences

\begin{abstract}
Objective: Online learning can be an effective learning approach, and provides one means to overcome scheduling conflicts for interprofessional education among students from multiple professions. One common topic for students from many health professions is helping patients contemplate and move towards their health behavior change (HBC). Change in a patient's health behavior can be an important outcome when examining chronic disease care, patient self-care and lifestyle management. Our objective was to develop and pilot an engaging online interprofessional education module focused on HBC by patients.

Design: Thirty-eight first-year health-professions students were introduced to HBC concepts using an online primer to motivational interviewing. This was followed by cases with questions where students were asked to provide $H B C$-consistent responses. An online discussion board facilitated students' participation and interaction, where they all could respond to case questions and to their peers. The discussion board was monitored by a faculty member skilled in the practice of HBC and another skilled in interprofessional education.
\end{abstract}

Findings: Students reported the course to be valuable and an acceptable way to begin learning new communication skills, and about other health-professions. Students' self-ratings of empathy and understanding of patients who do not readily commit to behavior change improved significantly from pre-module to post-module.

Conclusions: Online programming focused on HBC seems a feasible approach to interprofessional education, when designed carefully. Collaboration among interprofessional faculty may also harness expertise not necessarily available within one profession's silo.

Keywords: interprofessional education, online, motivational interviewing

\section{DESCRIPTION OF PROBLEM}

The World Health Organization defines interprofessional education (IPE) as when students from two or more professions learn with, about and from one another. ${ }^{1}$ Within this definition of IPE learning, it can be easiest to implement learning with one another (i.e., students listening to lectures together), but educators cannot leave out the more difficult implementation of students learning about and from one another; these may need more deliberate attention during IPE course planning. Simply said, IPE cannot be accomplished with only listening to lectures together; instead students need to be able to interact with one another and learn more about each other in the process.

One important outcome in healthcare is to affect changes in patient's health-related behaviors. This involves clinicians developing the patient's perspective on self-care, lifestyle and disease management, and may also involve interprofessional cooperation. Motivational Interviewing (MI) is one empiricallybased intervention towards health behavior change (HBC). MI

Corresponding author: Michael J. Peeters, PharmD, MEd University of Toledo

College of Pharmacy \& Pharmaceutical Sciences michael.peeters@utoledo.edu is a patient-centered intervention that seeks to elicit reasons, needs, and a desire for change from the patient. ${ }^{2}$ While students from a variety of professions can learn about MI together through a lecture, that is not all of interprofessional education. Instead using the World Health Organization's definition, IPE also needs students from more than one profession learning about and from one another. Within the United States, core competencies of interprofessional collaboration include the areas of roles/responsibilities, teams/teamwork, interprofessional communications, and values/ethics. ${ }^{1}$ In this perspective on IPE, none of these areas are course-content topics themselves, though any and all can be facilitated through various course content, such as HBC and MI.

While use of standardized patients is one effective active learning strategy for $\mathrm{MI}$ instruction, ${ }^{3}$ this strategy demands resources that are not always available. An interactive online learning strategy for $\mathrm{HBC}$ could require fewer resources and, importantly, it could offer an interactive interprofessional learning opportunity for learners in different professional programs with busy, conflicting curricula schedules. Revisiting IPE (learning with, from, and about) using an online approach can take deliberate focus in order to facilitate learning from and about. In prior IPE reports of online offerings, ${ }^{4,5}$ this has seemed a challenge. 
Interventions for $\mathrm{HBC}$ require a foundation in basic listening and communication skills to support the development of increasingly complex skills. ${ }^{2}$ The spirit of $\mathrm{Ml}$ involves interpersonal style using reflective listening, acceptance and affirmation; this spirit communicates four elements: collaboration, compassion, acceptance and evocation. As well, this spirit involves an underlying mindset within which clinicians use their clinical skills, and this mindset is considered foundational for every $\mathrm{Ml}$ conversation (as well as being the first stage in developing $\mathrm{Ml} \mathrm{skills}{ }^{6}$ ). Learning interventions for $\mathrm{HBC}$ can be classified into phases. The first phase, learning the communication style that $\mathrm{MI}$ refers to, is believed to be one critical component for Ml's efficacy; it was thought that online learning was amenable with this first phase. As well, empathy has been reported as an essential element of providers' communication style, and contributes to $\mathrm{Ml}^{\prime} \mathrm{s}$ spirit and positive outcomes. ${ }^{2}$ Instruction in the communication style underlying this spirit was the basis for the online module.

To our knowledge, HBC has not yet been reported in the literature for IPE. Herein, we describe an innovation to introduce interprofessional groups of first-year healthprofessions students to patient-centered health behavior communication via an online module.

\section{INNOVATION}

\section{Participants}

An Interprofessional Education Health Behavior Change (IPE$\mathrm{HBC}$ ) module was developed and offered as an elective to students from multiple health professions. It was in addition to required participation in standardized patient simulations. In this pilot module, 38 students from medicine ( $n=17$; first-year), nursing ( $n=1$; third-year), pharmacy ( $n=19$; first-year) and physician assistant $(n=1$; first-year) professions voluntarily chose to participate. These students were divided interprofessionally into four groups of 9 or 10 members. For most of these students, this was before any clinical disease state instruction in any of their other coursework.

\section{IPE-HBC Module}

This four week online course included: Week 1 - introduction, an overview of $\mathrm{MI}$ demonstrating its application through both text and video vignettes; Week 2 - brief video and case presentation on medication adherence with emphasis on engagement, active listening and reflective listening; Week 3 brief video and case presentation on smoking cessation with emphasis on working through ambivalence, identifying change talk and using reflections; and Week 4 - brief video and case presentation on physical activity with emphasis on eliciting change-talk and use of reflection (see Figure 1). Each case had 4-5 questions meant to elicit responses consistent with patient-centered communication within the spirit of MI (see Table 1).
As an introduction to HBC in Week 1, students completed the "Motivational Interviewing in Brief Consultations;" an online learning lecture available through the British Medical Journal [http://learning.bmj.com/learning/module-

intro/.html?moduleid=10051582]. The lecture focused on the four processes of $\mathrm{Ml}$ (i.e., engaging, focusing, evoking and planning) using text and video demonstrations of $\mathrm{MI}$ consistent skills, emphasizing a guiding-style of communication instead of a directive-style. It was self-paced and concluded with a self-assessment test (requiring $70 \%$ pass rate) and a certificate of completion that students were required to submit on Blackboard as evidence of task completion. This lecture was chosen for its availability and because it was developed by Stephen Rollnick, who together with William Miller initially developed and disseminated the $\mathrm{MI}$ approach to behavior change around the world. ${ }^{2}$ Module faculty felt that Dr. Rollnick's expertise in both the training and practice of $\mathrm{Ml}$ was evident in this learning experience. Importantly, this lecture was developed for a healthprofessions audience and provided video vignettes (with Dr. Rollnick appearing as the MI interviewer) demonstrating the MI processes and skills being described throughout the lecture.

In each of Weeks 2-4, a new written case vignette was introduced (Week 2: Medication Adherence, Week 3: Smoking Cessation, Week 4: Physical Activity). As well, a module faculty provided a brief summary video ( $<8 \mathrm{~min}$ ) of pertinent information previously covered in the Week 1 BMJ lecture. Each case was followed by a set of questions designed to elicit patient-centered responses in a guiding-style (see Figure 1). Each case addressed a common health behavior and all were created by one of the authors, based upon similar cases used during MI training workshops. The format of presenting cases followed by patient statements and then a set of questions was adapted from the Video Assessment of Simulated Encounters - Revised (VASE-R), an instrument with validation evidence in assessing MI-proficiency among practitioners who had undergone MI-training. ${ }^{7}$ While the VASE-R uses video presentations of simulated patients and asks learners to make Ml-informed responses to the standardized patient statements and situation, within this IPE-HBC module the VASE-R was not directly used. Instead, its format of providing a clinical context along with patient statements of concern and ambivalence about change were used to engage students in the perspective taking and patient-centered communication style needed to engage patients in health behavior centered discussions (Table 1).

The case vignettes, student responses and faculty feedback were accomplished in entirety using the discussion board feature of Blackboard Learn (Blackboard Inc., Washington DC; the University of Toledo's learning management system). 
Students went online at their convenience, read the case, other students' responses to the questions, and formulated their own responses. All students in their particular interprofessional group could see and respond to all posts by other students.

\section{Instructors}

Two faculty members, one skilled in $\mathrm{Ml}$ and one skilled in interprofessional practice monitored each of the four IPE-HBC groups. Faculty monitoring each discussion board did not respond to student comments individually, but instead responded at the end of the students' discussion. Faculty responded by affirming responses consistent with the spirit and practice of $\mathrm{MI}$ and interprofessional collaborative practice, providing constructive comments, posing thought-provoking questions to the group, and concretely linking student responses to the material presented in the online BMJ lecture. Consistent with the training philosophy of the facilitators, responses to learners were meant to be supportive. Awkwardly phrased or "slightly" paternalistic statements were not always highlighted, but that portion of the learner's statement consistent with a guiding-style was supported, while sometimes pointing out a better way to phrase a statement (Table 1).

\section{Outcome}

The health-professions literature suggests a significant decline in empathetic attitudes and behaviors through medical and postgraduate training. ${ }^{8}$ Students can be socialized to have negative views of non-adherent patients or those who appear to be purposefully unhealthy. As has been mentioned, empathy is thought to be one essential element of a guidingstyle of communication that is felt to contribute to the spirit of $\mathrm{MI}$ and positive health behavior outcomes. ${ }^{2}$ Thus, herein we used the Jefferson Scale of Empathy-Health Professions Student (JSE-HPS) version, ${ }^{9}$ before and after this module to assess students' overall attitudes related to empathizing with patients, which includes understanding each patient's experience and thinking like that patient.

\section{CRITICAL ANALYSIS}

This study was IRB-approved as exempt. The main quantitative assessment of this pilot was students' attitudes related to empathy. With internal consistencies of pre-module JSE-HPS $=0.8$ and post-module JSE-HPS $=0.7$, a paired-samples $t$-test was conducted to compare pre-module and post-module JSEHPS scores. There was a significant difference between the pre-module JSE-HPS (mean=103, standard deviation=13) and post-module JSE-HPS scores (mean=121, standard deviation=10; $p<0.001$; Cohen's $d=1.5$ ). Practical significance can be described by effect size, and at 1.5 it was considered to be very large by Cohen's interpretation. ${ }^{10}$ That is, practicallyspeaking these students appeared to substantially improve their attitudes toward empathy with this module. Similarly, this effect was also statistically significant using linear regression to analyze change with pre-module JSE-HPS regressed on post-module JSE-HPS $(p=0.004)$.

In students' module completion evaluations, they were asked to evaluate their most favorite and least favorite parts of the learning module experience. These responses were coded into categories. Most responses fell into five most favorite categories and four least favorite categories. Of most favorite parts to students' experiences, 32\% reported it enhanced their interviewing skills, $23 \%$ reported that it fit their schedule and was flexible, $13 \%$ reported reading the other student and facilitators comments, while $7 \%$ of students found the video demonstrations helpful, and $7 \%$ others reported liking the cases used. For the least favorite experiences, 35\% found some of the timing of due dates confusing, 27\% reported "not applicable" or "no problems", while $19 \%$ felt the discussion board felt forced and less natural then they would have liked.

Additionally, using an online module for IPE instruction had a further benefit. While It allowed learners from a variety of professional programs with different curricular schedules to more conveniently participate, ${ }^{4,5}$ its development and implementation notably required interprofessional faculty collaboration, and thus exposed learners to experts in the fields of both $\mathrm{HBC}$ and interprofessional collaborative practice.

We did not track other learning experiences for these students during the study timeframe and so we cannot rule out confounding variables having a role to play in this change in attitude towards empathy. However, we believe the learning module (with its clinical vignettes) influenced students' views on what would traditionally be seen as "resistant" or "nonadherent" patients. The students being asked to respond with a guiding-style of communication, rather than directivestyle, seeing how other students responded to the same scenario and receiving expert faculty feedback is believed to have contributed to a more empathetic, accepting and nonjudgmental perspectives. We also acknowledge that roleplaying and/or standardized patient interactions along with facilitator feedback is a gold standard for competence in developing communication skills and MI skills in particular; this introductory module did not offer that level of learner experience.

\section{NEXT STEPS}

Within our numerous health-professions programs, one challenge is to offer a more advanced module(s) for students. We envision an advanced module(s) that will have students develop actual MI skills with videos, standardized and actual patient interactions. Assessing fidelity to MI would be most appropriate at this next stage. With this small IPE module, we 
were able to overcome one common obstacle, scheduling, by using an online, asynchronous module for students to learn with, from and about each other's health professions.

In this educational research report, we have provided preliminary evidence suggesting an interprofessional approach to teaching health behavior change (with a focus on using a guiding-style, instead of directive-style for patientcentered communication) enhances attitudes towards empathy. Using our interprofessional colleagues with expertise in MI and IPE, we appear to have successfully created and implemented an online IPE module in which students from a number of health professions perceived it as helping them to learn with, from, and about one another.

Acknowledgements: We thank Mary Kay Smith, MD, for her direction and facilitation in bringing us together, and the support received in developing this mod

Disclosures: This project was funded in part by the Ohio MEDTAPP Healthcare Access grant.

\section{REFERENCES}

1. Interprofessional Education Collaborative. Core competencies for interprofessional collaborative practice: 2016 update. Washington, DC:

Interprofessional Education Collaborative; 2016. https://www.ipecollaborative.org/resources.html. Accessed February 27, 2018.

2. Miller WR, Rollnick S. Motivational Interviewing: Helping People Change. New York, NY: Guilford Press; 2013.

3. Lupu AM, Stewart AL, O'Neil C. Comparison of active-learning strategies for motivational interviewing skills, knowledge, and confidence in first-year pharmacy students. Am J Pharm Educ, 2012;76(2): Article 28.

4. Dow AW, Boling PA, Lockeman KS, Mazmanian PE, Feldman M, DiazGranados, et al. Training and assessing interprofessional virtual teams using a web-based case system. Acad Med. 2016; 91(1):120126.

5. Poirier TI, Devraj R, Blankson F, Xin $\mathrm{H}$. Interprofessional online global health course. Am J Pharm Educ. 2016; 80(9): Article 155.

6. Miller WR, Moyers TB. Eight stages in learning motivational interviewing. J Teach Addict. 2006; 5(1): 13-27.

7. Rosengren DB, Hartzler B, Baer JS, Wells EA, Dunn $\mathrm{CW}$. The video assessment of simulated encountersrevised (VASE-R): Reliability and validity of a revised measure of motivational interviewing skills. Drug Alcohol Depend. 2008; 97(1-2):130-138.
8. Kelly J. When I say...empathy. Med Educ. 2017; 51(6):573-574.

9. Hojat M, Gonnella JS, Maxwell K. Jefferson Scales of Empathy (JSE): Professional Manual \& User's Guide. Philadelphia PA: Jefferson Medical College; 2009.

10. Peeters MJ. Practical significance: Moving beyond statistical significance. Curr Pharm Teach Learn. 2016;8(1):83-89. 
Table 1. Abbreviated Sample of Case and its Discussion Thread.

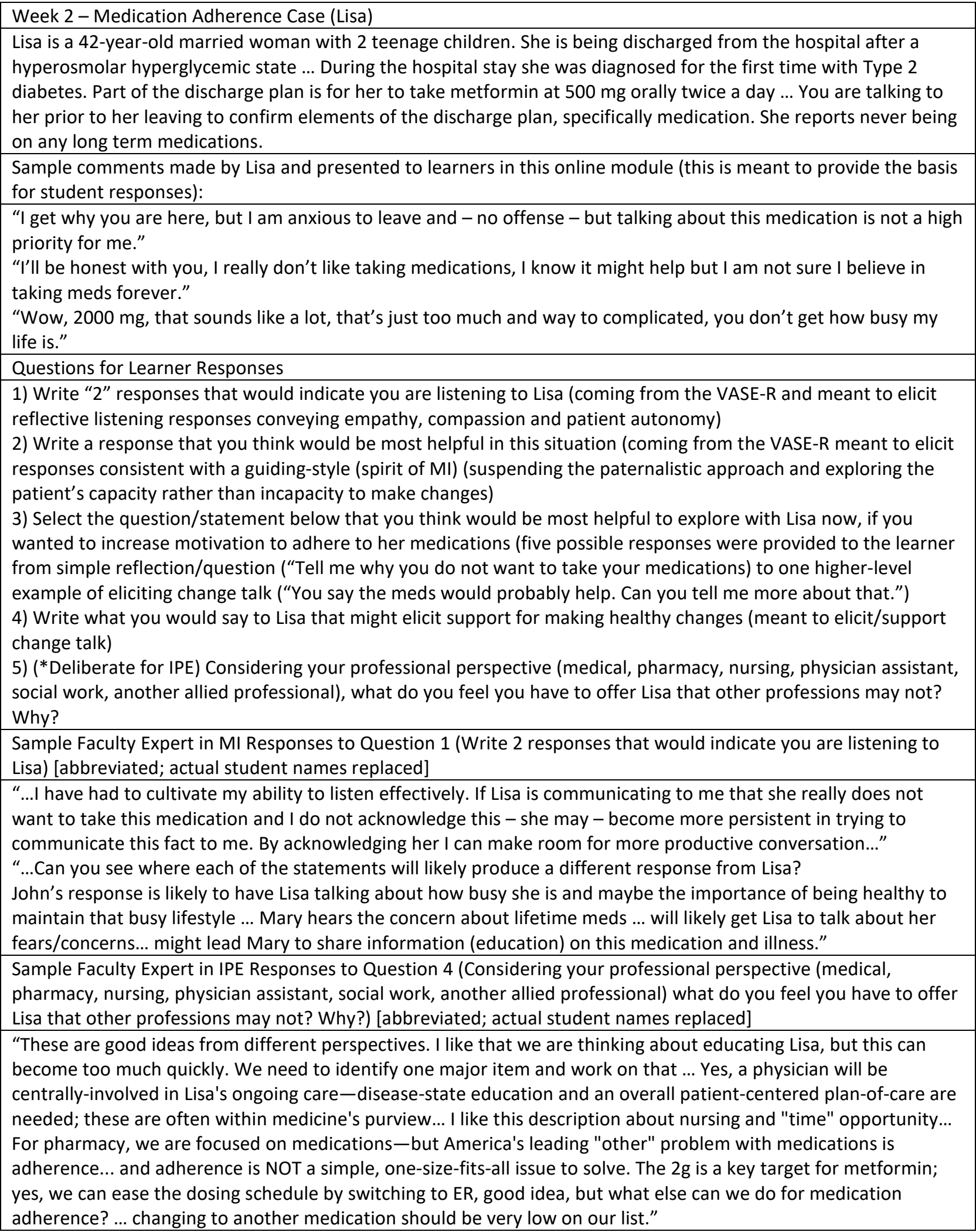


Figure 1. Diagram of IPE-HBC Course Content and Procedure

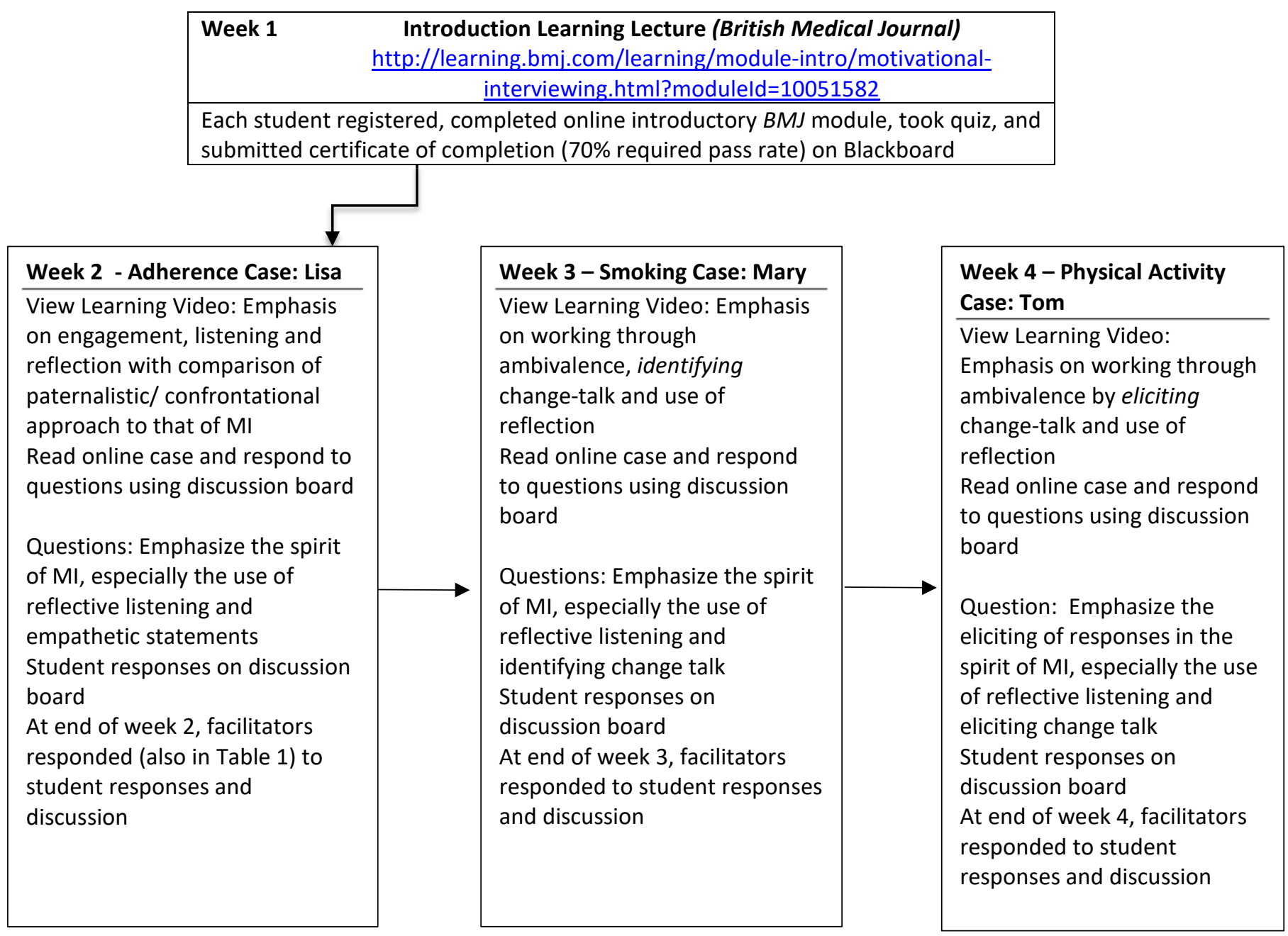

